\title{
Severe maternal morbidity in Canada, 1991-2001
}

\author{
Shi Wu Wen, Ling Huang, Robert Liston, Maureen Heaman, Tom Baskett, I.D. Rusen, \\ K.S. Joseph, Michael S. Kramer; for the Maternal Health Study Group, Canadian Perinatal \\ Surveillance System
}

An abridged version of this article appeared in the Sept. 27, 2005, issue of CMAJ.

Abstract

Background: Although death rates are often used to monitor the quality of health care, in industrialized countries maternal deaths have become rare. Severe maternal morbidity has therefore been proposed as a supplementary indicator for surveillance of the quality of maternity care. Our purpose in this study was to describe severe maternal morbidity in Canada over a 10-year period, among women with or without major pre-existing conditions.

Methods: We carried out a retrospective cohort study of severe maternal morbidity involving 2548824 women who gave birth in Canadian hospitals between 1991 and 2000. Thirteen conditions that may threaten the life of the mother (e.g., eclampsia) and 11 major pre-existing chronic conditions (e.g., diabetes) that could be identified from diagnostic codes were noted.

Results: The overall rate of severe maternal morbidity was 4.38 per 1000 deliveries. The fatality rate among these women was 158 times that of the entire sample. Rates of venous thromboembolism, uterine rupture, adult respiratory distress syndrome, pulmonary edema, myocardial infarction, severe postpartum hemorrhage requiring hysterectomy, and assisted ventilation increased substantially from 1991 to 2000. The presence of major pre-existing conditions increased the risk of severe maternal morbidity to 6-fold.

Interpretation: Severe maternal morbidity occurs in about 1 of 250 deliveries in Canada, with marked recent increases in certain morbid conditions such as pulmonary edema, myocardial infarction, hemorrhage requiring hysterectomy, and the use of assisted ventilation.

Cite this article as CMAJ 2005;173(7). DOI:10.1503/cmaj.045156

M aternal mortality is an important measure of quality in maternity care..$^{1-3}$ However, maternal death rates are now so low in industrialized countries that use of the measure could cause complacency. Conversely, unstable statistics based on small numbers and resultant misinterpretation could lead to recommendations based on unusual events of little relevance to the care of most pregnant women. ${ }^{4}$ Severe maternal morbidity has therefore been proposed as a supplementary indicator for monitoring the quality of maternity care. ${ }^{1,2,4,5}$
Severe morbidity appears along a health continuum that extends from normal pregnancy and delivery to organ failure and maternal death. In maternity care, severe morbidity and death betoken and therefore measure similar medical problems, but the incidence of severe morbidity is much higher. Well-established indicators of maternal morbidity based on a large body of data could be used to portray the magnitude of problems in maternal care and to improve its quality; for example, an excessively high rate for an indicator in a particular jurisdiction may warrant a local audit and appraisal of standard care.

A few studies have examined specific severe maternal morbidities such as amniotic-fluid embolism, ${ }^{6}$ adult respiratory distress syndrome, ${ }^{7}$ anesthesia, ${ }^{8}$ eclampsia ${ }^{9}$ and uterine rupture. ${ }^{10}$ Information in the literature on comprehensive measures of severe maternal morbidity generated from a large population is sparse. Our objective in this study was to describe the occurrence and temporal trends in Canada of severe maternal morbidity among women with or without major pre-existing conditions, using information on hospital discharges compiled by the Canadian Institute for Health Information (CIHI).

\section{Methods}

We used hospital admission and separation records collected by CIHI for fiscal years 1991/92 through 2000/01. Data for women admitted to hospital for obstetric delivery were abstracted by a combination of case-mix group, diagnostic and procedure codes defining their deliveries. ${ }^{11}$ During the study period, CIHI coded diagnoses according to the International Classification of Diseases, 9th Revision (ICD-9) ${ }^{12}$ and coded procedures according to the Canadian Classification of Diagnostic, Therapeutic, and Surgical Procedures (CCP) ${ }^{13}$ Complete data on about $70 \%$ of all obstetric deliveries in Canada were recorded by CIHI during the 10-year study period. Because data on deliveries in the provinces of Quebec, Manitoba and Nova Scotia were incomplete, they were excluded from our analysis.

The conditions defined as severe maternal morbidities and assessed in this analysis comprised venous thromboembolism, eclampsia, pulmonary, cardiac or central-nervous-system complications of anesthesia, cerebrovascular disorders in the puerperium (including intracranial venous sinus thrombosis), uterine rupture, adult respiratory distress syndrome, pulmonary edema, myocar- 
dial infarction, acute renal failure after labour and delivery, cardiac arrest or failure or cerebral anoxia after obstetric surgery, severe postpartum hemorrhage requiring hysterectomy or transfusion, and the need for assisted ventilation. The ICD-9 and CCP codes for these conditions are listed in Appendix 1. The criteria for selecting severe maternal morbidity were those "near miss" conditions with potential to cause maternal death that could be reliably identified from the CIHI hospital-discharge database. The decisions were based on both the clinical importance of these conditions and the results of our exploration of the strengths and weaknesses of the database. For example, amniotic-fluid embolism is well-known as a cause of maternal death; but because our preliminary analysis showed that the case-fatality rate for this condition in the database was only about 30\% (much below the rate of $>60 \%$ reported in the literature), we did not include it in the final list. Because preliminary analysis of the data identified a coding error for myocardial infarction in records for obstetric deliveries in Saskatchewan, we excluded all Saskatchewan deliveries from our calculation of myocardial infarction rates. In calculating total maternal morbidity rates, we included Saskatchewan deliveries but not myocardial infarctions, which we reclassified as noncases.

We also assessed the effect on the occurrence and severity of maternal morbidity of major pre-existing chronic diseases, including systemic lupus erythematosus, cystic fibrosis, chronic renal disease, essential hypertension, diabetes mellitus with and without (renal and other) complications, primary and secondary pulmonary hypertension, and congenital, chronic rheumatic and ischemic heart disease. The ICD-9 codes for pre-existing conditions are listed in Appendix 2.

To measure the overall burden of severe maternal morbidity,

\begin{tabular}{|c|c|c|c|}
\hline Maternal morbidity & $\begin{array}{l}\text { No. of } \\
\text { cases }\end{array}$ & $\begin{array}{c}\text { Rate per } 1000 \\
\text { deliveries }(95 \% \mathrm{Cl})\end{array}$ & $\begin{array}{l}\text { Deaths } \\
\text { (\% of cases) }\end{array}$ \\
\hline $\begin{array}{l}\text { Cardiac arrest or failure or cerebral anoxia } \\
\text { after obstetric surgery }\end{array}$ & 2677 & $1.05(1.01-1.09)$ & 37 (1.4) \\
\hline Postpartum hemorrhage requiring transfusion & 2317 & $0.91(0.87-0.95)$ & $7 \quad(0.3)$ \\
\hline Uterine rupture & 1898 & $0.74(0.71-0.78)$ & $4 \quad(0.2)$ \\
\hline Pulmonary, cardiac or central-nervous-system & 1246 & $0.49(0.46-0.52)$ & $2 \quad(0.2)$ \\
\hline Eclampsia & 973 & $0.38(0.36-0.41)$ & $4 \quad(0.4)$ \\
\hline Postpartum hemorrhage requiring hysterectomy & 892 & $0.35(0.33-0.37)$ & 14 (1.6) \\
\hline $\begin{array}{l}\text { Cerebrovascular disorders in the puerperium, } \\
\text { including intracranial venous sinus thrombosis }\end{array}$ & 412 & $0.16(0.15-0.18)$ & 19 (4.6) \\
\hline Pulmonary edema & 402 & $0.16(0.14-0.17)$ & $3 \quad(0.7)$ \\
\hline Need for assisted ventilation & 387 & $0.15(0.14-0.17)$ & $29 \quad(7.5)$ \\
\hline Venous thromboembolism & 334 & $0.13(0.12-0.15)$ & $14 \quad(4.2)$ \\
\hline Acute renal failure after delivery & 219 & $0.09(0.07-0.10)$ & $5 \quad(2.3)$ \\
\hline Adult respiratory distress syndrome & 205 & $0.08(0.07-0.09)$ & $11 \quad(5.4)$ \\
\hline Myocardial infarction & 31 & $0.01(0.01-0.02)$ & $2 \quad(6.5)$ \\
\hline \multicolumn{4}{|l|}{ Summary of women with morbidities $\dagger$} \\
\hline 1 morbid condition only & 10307 & $4.04(3.96-4.12)$ & $41 \quad(0.4)$ \\
\hline 2 morbid conditions & 621 & $0.25(0.23-0.27)$ & $26 \quad(4.2)$ \\
\hline 3 or more morbidities & 138 & $0.06(0.05-0.07)$ & $17(12.3)$ \\
\hline Totals, women with $\geq 1$ morbidity & 11066 & $4.38(4.30-4.46)$ & $84 \quad(0.8)$ \\
\hline
\end{tabular}

*Excluding Quebec, Manitoba and Nova Scotia. †Severe maternal morbidity, as defined in this study. we calculated the rate for women with any of the severe maternal morbidity conditions. To measure the severity of the chosen indicators, we calculated in-hospital death rates among women with various severe maternal morbidity conditions and compared these rates with the in-hospital death rate among all women who gave birth during the study period. To assess if we missed some important severe maternal morbidity conditions in our list, we examined the ICD-9 codes for conditions in women who died in hospital that were not included by our definition of severe maternal morbidity. To assess possible increases in the rates of these conditions, we compared the rates of severe maternal morbidity in 1991-1993 with those in 1998-2000. To highlight changes over time, we also graphed yearly rates for selected indicators. To assess the influence of demographic changes, we conducted additional analyses of temporal trends, adjusting for maternal age, multifetal pregnancy and previous cesarean delivery.

We analyzed the rates of pre-existing conditions, in-hospital death and severe maternal morbidity to search for relations. To assess to what extent the incidence and severity of maternal morbidity are affected by pre-existing chronic diseases, we conducted supplementary analyses of the occurrence and in-hospital deaths in women with various severe maternal morbidity conditions, after excluding women with pre-existing conditions.

\section{Results}

The CIHI database had records for a total of 2548824 obstetric deliveries (a number that excludes deliveries in Quebec, Manitoba and Nova Scotia) during the 10-year period of this study. Among these, we identified 11066 cases with at least 1 condition defined by us as a severe morbidity, yielding an overall rate of 4.38 severe maternal morbidities per 1000 deliveries (Table 1). The rate of in-hospital death ranged from $7.5 \%$ among women who required assisted ventilation to $0.16 \%$ among those with anesthesia complications. The rate was far higher among women with severe maternal morbidity than in the overall study sample.

Some of the women who died in hospital had conditions not included in our list of severe maternal morbidities (Table 2).${ }^{14}$ When the entire sample population was considered, cardiac arrest unrelated to obstetric surgery was the condition coded most frequently; but when data on women who died who had no study-defined morbidities were analyzed, the code most frequently seen was that for injury or poisoning.

Ten-year trends for 6 of the severe maternal morbidities considered are shown in Table 3 and Fig. 1. Rates of 
venous thromboembolism, uterine rupture, adult respiratory distress syndrome, pulmonary edema, myocardial infarction, severe postpartum hemorrhage requiring hysterectomy, and need for assisted ventilation increased substantially ( $\geq 50 \%$ or more) over the 10 -year period; that for cerebrovascular disorders in the puerperium increased moderately $(<50 \%)$; that for severe postpartum hemorrhage requiring transfusion declined (Fig. 1); and those for eclampsia, anesthesia complications, acute renal failure after delivery, cardiac arrest or failure or cerebral anoxia after obstetric surgery, and overall severe maternal morbidity showed no consistent trends. Adjustment for maternal age, multifetal pregnancy and previous cesarean delivery did not change the results (data available upon request).

The database included 9352 cases with 1 or more of the specified pre-existing chronic diseases, yielding a proportion in our study population of 3.67 per 1000 deliveries (Table 4). Their rate of in-hospital death was $0.14 \%$, higher than that among women without pre-existing chronic diseases but substantially lower than that among those with severe maternal morbidity.

The risk of severe maternal morbidity among women with pre-existing chronic diseases was increased substantially to 5.8 -fold the risk among those without pre-existing conditions (Table 5). Increases for individual comorbidities ranged from only about 1.5 - to 2 -fold (uterine rupture, adult respiratory distress syndrome and postpartum hemorrhage requiring hysterectomy or transfusion) up to 30-fold (pulmonary edema) and 50-fold (myocardial infarction). Excluding women with at least 1 pre-existing chronic condition reduced the risk of in-hospital death among women with severe maternal morbidity conditions only slightly (Table 6).

\section{Table 2: Conditions* of the women who died in hospital that} were not defined in this study as severe maternal morbidities

\begin{tabular}{|c|c|c|}
\hline \multirow[b]{2}{*}{ Condition } & \multicolumn{2}{|c|}{ No. $(\%)$ of woment } \\
\hline & $\begin{array}{l}\geq 1 \text { morbidity } \neq \\
\quad n=84\end{array}$ & $\begin{array}{c}\text { No morbidities } \neq \\
n=39\end{array}$ \\
\hline $\begin{array}{l}\text { Cardiac arrest unrelated to obstetric } \\
\text { surgery }\end{array}$ & $24(29)$ & $5(13)$ \\
\hline Injury or poisoning & $23(27)$ & $26(67)$ \\
\hline Pre-eclampsia & $20(24)$ & $4(10)$ \\
\hline Obstetric shock & $18(21)$ & $0-$ \\
\hline Previous cesarean section & 17 (20) & $0-$ \\
\hline Anemia & $17(20)$ & $0-$ \\
\hline $\begin{array}{l}\text { Pre-existing cardiovascular disease } \\
\text { complicating pregnancy }\end{array}$ & 13 (15) & $0-$ \\
\hline Breech presentation & 11 (13) & $0-$ \\
\hline Defibrination syndrome & $10(12)$ & $0-$ \\
\hline Infection & $5 \quad(6)$ & $0-$ \\
\hline Cancer & $4 \quad(5)$ & $13(33)$ \\
\hline
\end{tabular}

*Conditions were identified from patients' charts by ICD-9 codes. +Because patients may have more than 1 diagnosis apiece, numbers do not add up. ¥Severe maternal morbidity as defined in this study, concurrent with the condition listed here.

\section{Interpretation}

Our study provides an estimate of the incidence of and temporal trends in severe maternal morbidity, based on an analysis of the hospital admission and separation records collected by CIHI. This analysis yielded a rate of 4.38 per 1000 deliveries for severe maternal morbidity in the general populace. We also observed large increases during the last decade in the rates of venous thromboembolism, uterine rupture, adult respiratory distress syndrome, pulmonary edema, myocardial infarction and severe postpartum hemorrhage requiring hysterectomy. The use of assisted ventilation increased as well. No change was observed in rates of eclampsia, anesthesia complications, acute renal failure after delivery, cardiac arrest or failure or cerebral anoxia after obstetric surgery, or overall severe maternal morbidity.

Our study also provides an estimate of the incidence of major pre-existing chronic diseases among pregnant women: 3.67 per 1000 deliveries. The presence of major preexisting conditions predisposes pregnant women to an increased risk of severe maternal morbidity and mortality. (It should be recognized that it is sometimes difficult for one to separate chronic diseases from conditions that occur during pregnancy [e.g., thromboembolic disease] using ICD-9 codes.) Regardless of pre-existing chronic conditions, severe maternal morbidity occurs mostly among young and otherwise healthy women, because excluding women with pre-existing chronic diseases from our analysis reduced the overall risk of severe maternal morbidity only slightly.

Previous studies have estimated the incidence of individual causes of severe maternal morbidity. ${ }^{7-10}$ Using medical charts for obstetric deliveries from the Medical Sciences Center of the University of Arkansas, Catanzarite and colleagues $^{7}$ estimated a rate of acute respiratory distress syndrome of 0.16 per 1000 deliveries. This rate is almost double that observed in our population (0.08 per 1000), probably owing to the high-risk obstetric population served at the US centre. Studies in Nova Scotia found that the rate of eclampsia was 0.27 per 1000 deliveries $^{9}$ and the rate of uterine rupture, 0.34 per $1000,{ }^{10}$ both lower than the rates observed in our study ( 0.38 per 1000 and 0.74 per 1000, respectively). The Nova Scotia data are more comprehensive and contain more clinical details, which may improve the accuracy of the diagnoses. Geographic variation in outcomes secondary to regional variations in practice is another possibility, one that should be explored in future studies.

Our study used administrative data, which are prone to a certain degree of coding error. ${ }^{12}$ Particular attention should be paid to this when rare conditions (such as severe maternal morbidity) are involved, because the effect of coding errors is greater. In our preliminary analysis, for example, we found that the rate of myocardial infarction during pregnancy or delivery decreased markedly (from 0.26 per 1000 deliveries in 1991 to 0.03 per 1000 in 2000). This observation is counterintuitive and is not supported by changes in 
Table 3: Rates in Canada* of severe maternal morbidity during 19911993 and 1998-2000

\begin{tabular}{lccc}
\hline & \multicolumn{2}{c}{ Rate per 1000 deliveries } & Relative risk $\dagger$ \\
\cline { 2 - 3 } Maternal morbidity & $1991-1993$ & $1998-2000$ & $(95 \% \mathrm{Cl})$ \\
\hline Venus thromboembolism & 0.12 & 0.20 & $1.73(1.33-2.24)$ \\
Eclampsia & 0.38 & 0.33 & $0.88(0.75-1.05)$ \\
Anesthesia complications & 0.48 & 0.51 & $1.06(0.92-1.22)$ \\
Cerebrovascular disorders & 0.14 & 0.19 & $1.42(1.10-1.82)$ \\
Uterine rupture & 0.58 & 0.92 & $1.60(1.42-1.81)$ \\
Adult respiratory distress syndrome & 0.07 & 0.10 & $1.50(1.05-2.13)$ \\
Pulmonary edema & 0.10 & 0.22 & $2.09(1.60-2.72)$ \\
Myocardial infarction & 0.00 & 0.02 & $3.70(1.21-11.35)$ \\
Acute renal failure after delivery & 0.07 & 0.09 & $1.20(0.84-1.71)$ \\
Cardiac arrest or failure or cerebral & & & \\
anoxia after obstetric surgery & 1.05 & 0.96 & $0.91(0.82-1.00)$ \\
Hemorrhage & & & \\
$\quad$ Requiring hysterectomy & 0.26 & 0.46 & $1.76(1.48-2.08)$ \\
$\quad$ Requiring transfusion & 1.27 & 0.63 & $0.50(0.44-0.55)$ \\
Assisted ventilation & 0.10 & 0.24 & $2.47(1.89-3.23)$ \\
\hline Summary of women with morbidities & & & \\
$\quad 1$ morbidity only & 4.39 & 4.25 & $1.00(0.94-1.05)$ \\
2 morbidities & 0.20 & 0.37 & $2.12(1.75-2.57)$ \\
3 or more morbidities & 0.04 & 0.08 & $1.90(1.23-2.92)$ \\
All with $\geq 1$ maternal morbidity & 4.63 & 4.62 & $1.00(0.95-1.04)$ \\
\hline
\end{tabular}

*Excluding Quebec, Manitoba and Nova Scotia.

†The 1991-1993 period was used as the reference.

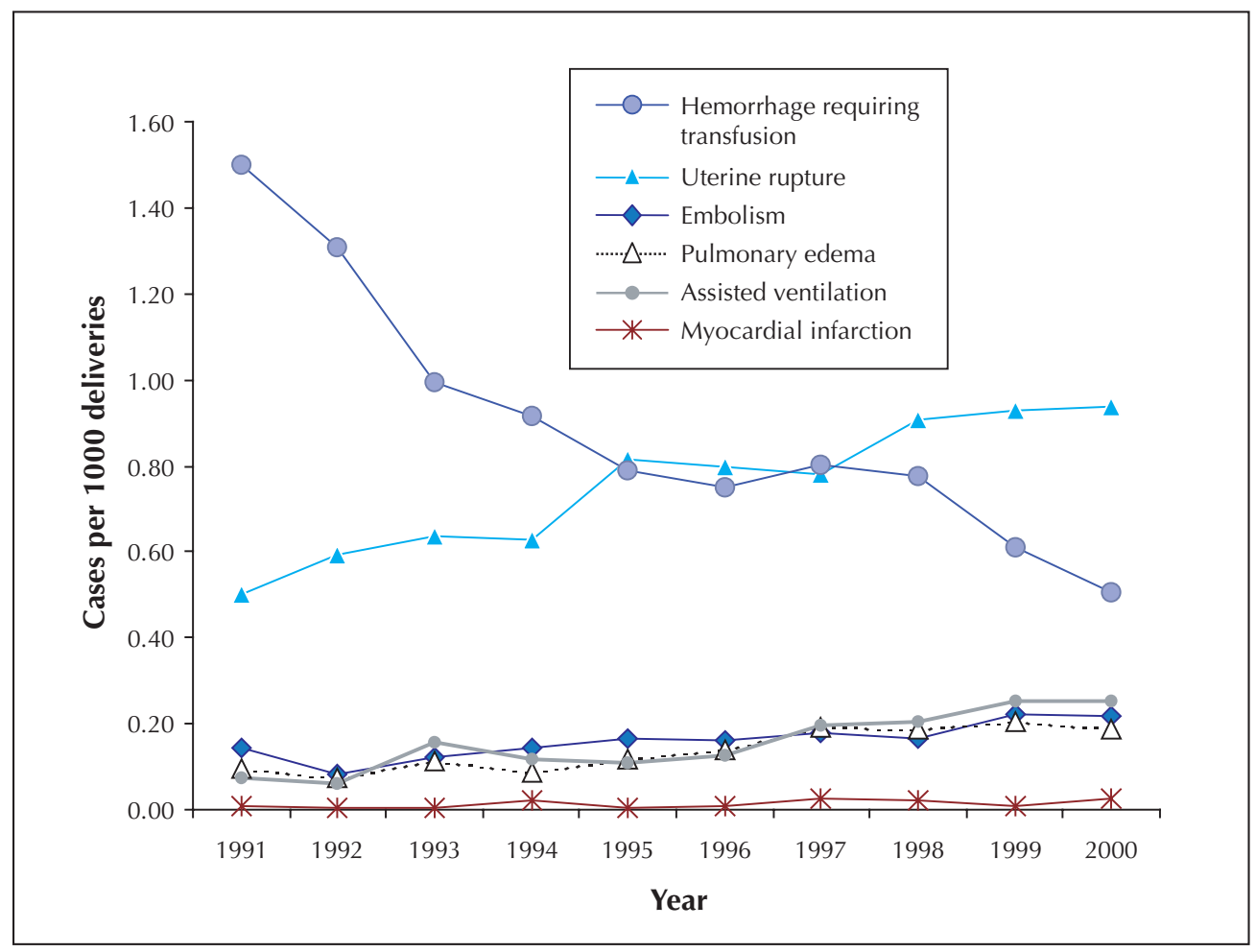

Fig. 1: Temporal trends in specific types of severe maternal morbidity in Canada, for the fiscal years 1991/92 through 2000/01. 
population demography (e.g., the increase in maternal age). Further exploration of this anomalous temporal pattern revealed that almost all of the myocardial infarction cases in 1991-1996 (342 cases of 353 in Canada) occurred in Saskatchewan, which accounts for only about $3 \%$ of the country's population. No myocardial infarction case was reported in that province after 1996. We therefore reclassified all myocardial infarction cases in Saskatchewan as non-cases, after which an increase to 3.5 -fold in the rate of myocardial infarction in Canada during the 10 years of study was observed instead. Amniotic-fluid embolism is a severe maternal morbidity with a high case-fatality rate. ${ }^{6}$ In the preliminary analysis we found that the incidence of amniotic-fluid embolism based on ICD-9 codes in CIHI data was about twice the rates seen in other clinical settings, whereas the case-fatality rate was only about half that reported in the literature. ${ }^{6}$ These abnormalities cast doubt on the ICD-9 coding of this condition in CIHI data. Since we could not find the sources of coding errors and the means to correct them, we have not presented our study data on amnioticfluid embolism.

In summary, we found that severe maternal morbidity occurs in about 1 of 250 deliveries in Canada. Considerable increases have occurred over the past decade in rates of venous thromboembolism, uterine rupture, adult respiratory distress syndrome, pulmonary edema, myocardial infarction, severe postpartum hemorrhage requiring hysterectomy, and the need for assisted ventilation. Because adjustment for maternal age, multifetal pregnancy and previous cesarean delivery did not change the overall results, these changes are unlikely to be caused by demographic trends; investigational attention should be paid to the reasons for these increases.

Severe maternal morbidity is associated with increased risk of maternal death and may result in substantial costs to the health care system and to society. Policy-makers and care

\begin{tabular}{|c|c|c|c|}
\hline Pre-existing condition & Cases & $\begin{array}{l}\text { Per } 1000 \\
\text { deliveries }\end{array}$ & $\begin{array}{l}\text { Deaths } \\
\text { (\% of cases) }\end{array}$ \\
\hline Diabetes mellitus & 3285 & 1.29 & $2(0.06)$ \\
\hline Congenital heart disease & 2215 & 0.87 & $3(0.14)$ \\
\hline Essential hypertension & 1239 & 0.49 & $2(0.16)$ \\
\hline Systemic lupus erythematosus & 1155 & 0.45 & $3(0.25)$ \\
\hline Chronic rheumatic heart disease & 655 & 0.26 & 0 \\
\hline Chronic renal disease & 552 & 0.22 & $2(0.36)$ \\
\hline Diabetes with complications & 358 & 0.14 & 0 \\
\hline Ischemic heart disease & 71 & 0.02 & $1(1.41)$ \\
\hline Cystic fibrosis & 75 & 0.03 & 0 \\
\hline Pulmonary hypertension & 69 & 0.03 & $3(4.34)$ \\
\hline $\begin{array}{l}\text { Totals, women with } \geq 1 \\
\text { pre-existing condition }\end{array}$ & 9352 & 3.67 & $13(0.14)$ \\
\hline
\end{tabular}

*Excluding Quebec, Manitoba and Nova Scotia.
Table 5: Rates of severe maternal morbidity in Canada* among women with or without major pre-existing conditions, 1991-2001

\begin{tabular}{|c|c|c|c|}
\hline \multirow{3}{*}{$\frac{\text { Maternal morbidity }}{\text { Venus thromboembolism }}$} & \multicolumn{3}{|c|}{$\begin{array}{l}\text { No. of women } \\
\text { (rate per } 1000 \text { deliveries) }\end{array}$} \\
\hline & \multicolumn{2}{|c|}{$\begin{array}{l}\geq 1 \text { pre-existing } \\
\text { condition }\end{array}$} & $\begin{array}{l}\text { No pre-existing } \\
\text { conditions }\end{array}$ \\
\hline & 15 & $(1.60)$ & $319(0.13)$ \\
\hline Eclampsia & 17 & $(1.82)$ & $956(0.38)$ \\
\hline $\begin{array}{l}\text { Pulmonary, cardiac or CNS } \\
\text { complications of anesthesia }\end{array}$ & 20 & $(2.14)$ & $1226(0.48)$ \\
\hline $\begin{array}{l}\text { Cerebrovascular disorders in } \\
\text { the puerperium } \dagger\end{array}$ & 20 & $(2.14)$ & $392(0.15)$ \\
\hline Uterine rupture & 19 & $(2.03)$ & 1879 (0.74) \\
\hline Adult respiratory distress syndrome & 12 & $(1.28)$ & $193 \quad(0.08)$ \\
\hline Pulmonary edema & 46 & $(4.92)$ & $356 \quad(0.14)$ \\
\hline Myocardial infarction & 6 & $(0.64)$ & $25(0.01)$ \\
\hline Acute renal failure after delivery & 8 & $(0.84)$ & $211 \quad(0.08)$ \\
\hline $\begin{array}{l}\text { Cardiac arrest or failure or cerebral } \\
\text { anoxia after obstetric surgery }\end{array}$ & 68 & $(7.27)$ & 2609 (1.03) \\
\hline \multicolumn{4}{|l|}{ Postpartum hemorrhage } \\
\hline Requiring hysterectomy & 8 & $(0.86)$ & $884 \quad(0.35)$ \\
\hline Requiring transfusion & 12 & $(1.28)$ & $2305 \quad(0.91)$ \\
\hline Need for assisted ventilation & 20 & $(2.14)$ & $367(0.14)$ \\
\hline Total, women with $\geq 1$ morbidity & 232 & (24.34) & $10834 \quad(4.27)$ \\
\hline
\end{tabular}

Note: $\mathrm{CNS}=$ central nervous system.

*Excluding Quebec, Manitoba and Nova Scotia.

tIncluding intracranial venous sinus thrombosis.

Table 6: In-hospital death rates in Canada* among women with severe maternal morbidity but no major pre-existing conditions, 1991-2001

\begin{tabular}{lrrl}
\hline Morbidity & $\begin{array}{r}\text { No. of } \\
\text { cases }\end{array}$ & $\begin{array}{c}\text { Deaths } \\
\text { (\% of cases) }\end{array}$ \\
\hline $\begin{array}{l}\text { Venous thromboembolism } \\
\text { Eclampsia }\end{array}$ & 956 & $10(3.13)$ \\
Pulmonary, cardiac or central-nervous-system & & & $(0.42)$ \\
complications of anesthesia & 1226 & 1 & $(0.08)$ \\
Cerebrovascular disorders in the puerperium & & & \\
including intracranial venous sinus thrombosis & 392 & 16 & $(4.08)$ \\
Uterine rupture & 1879 & 4 & $(0.21)$ \\
Adult respiratory distress syndrome & 193 & $11(6.04)$ \\
Pulmonary edema & 356 & $3(0.84)$ \\
Myocardial infarction & 25 & $2(8.00)$ \\
Acute renal failure after delivery & 211 & $5(2.37)$ \\
Cardiac arrest or failure or cerebral anoxia & & & \\
after obstetric surgery & 2609 & $33(1.26)$ \\
Postpartum hemorrhage & & & \\
$\quad$ Requiring hysterectomy & 884 & $13(1.47)$ \\
$\quad$ Requiring transfusion & 2305 & $7(0.30)$ \\
Need for assisted ventilation & 367 & $27(7.36)$ \\
\hline Women with $\geq 1$ maternal morbidity & 10834 & $74(0.68)$ \\
All women who gave birth & 2540533 & $111(0.004)$ \\
\hline
\end{tabular}

*Excluding Quebec, Manitoba and Nova Scotia. 
providers should pay attention to this major public-health issue and devise interventions to prevent these "near miss" conditions or reduce their severity. We also found that the presence of major pre-existing chronic disease predisposes pregnant women to severe maternal morbidity. Finally, a large proportion of women who died in hospital, including those who had at least 1 condition meeting our criteria for severe maternal morbidity, had ICD-9 codes for conditions unrelated to pregnancy, such as injury or poisoning, cardiac arrest unrelated to obstetric surgery, and cancer, underlining the importance of injury prevention and appropriate treatment of pre-existing conditions in pregnant women.

This article has been peer reviewed.

From the OMNI Research Group, Department of Obstetrics and Gynaecology, Ottawa Health Research Institute, and the Clinical Epidemiology Program, Department of Epidemiology and Community Medicine, University of Ottawa (Wen, Rusen); the Health Surveillance and Epidemiology Division, Centre for Healthy Human Development, Health Canada (Huang), Ottawa, Ont.; the Department of Obstetrics and Gynaecology, University of British Columbia, Vancouver, BC (Liston); the Faculty of Nursing, University of Manitoba, Winnipeg, Man. (Heaman); the Department of Obstetrics and Gynaecology (Baskett, Joseph) and Perinatal Epidemiology Research Unit, Department of Pediatrics (Joseph), Dalhousie University, Halifax, NS; and the Departments of Pediatrics and of Epidemiology and Biostatistics, McGill University, Montréal, Que. (Kramer).

Competing interests: None declared.

Contributors: Shi Wu Wen contributed to the study concept and desion and the data analysis and interpretation, and drafted the manuscript. Ling Huang contributed to the drafting of the manuscript and did data analysis and interpretation. Robert Liston helped interpret the data and revised the manuscript. Maureen Heaman and Tom Baskett contributed to the concept and design of the study, interpretation of the data and revision of the manuscript. I.D. Rusen was active in data interpretation and manuscript revision. K.S. Joseph's and Michael Kramer's contributions included study concept and design, statistical expertise, data interpretation and manuscript revision.

Acknowledgements: We thank the people at the Canadian Institute for Health Information, who gave us access to their data files. This study was conducted under the auspices of the Canadian Perinatal Surveillance System.

Shi Wu Wen, Maureen Heaman, K.S. Joseph and Michael Kramer are recipients of Career Investigator Awards from the Canadian Institutes of Health Research.

\section{References}

1. Baskett TF, Sternadel J. Maternal intensive care and near-miss mortality in obstetrics. Br 7 Obstet Gynaecol 1998;105:981-4.

2. Mantel GD, Buchmann E, Rees H, Pattinson RC. Severe acute maternal morbidity: a pilot study of a definition for a near-miss. Br 7 Obstet Gynaecol 1998;105:985-90.

3. Hibbard BM, Anderson MM, Drife JO, Tighe JR, Gordon G, Willatts S, et al, for the Department of Health, Welsh Office; Scottish Home and Health Department; Department of Health and Social Services, Northern Ireland. Report on confidential enquiries into maternal deaths in the United Kingdom 1991-1993. London (UK): Her Majesty's Stationery Office; 1996.

4. Drife JO. Maternal "near miss" reports? BMF 1993;307:1087-8.

5. Bewley S, Greighton S. "Near miss" obstetric enquiry. 7 Obstet Gynaecol 1997; 17:26-9.

6. Gilbert WM, Danielsen B. Amniotic fluid embolism: decreased mortality in a population-based study. Obstet Gynecol 1999;93:973-7.

7. Catanzarite V, Willms D, Wong D, Landers C, Cousins L, Schrimmer D. Acute respiratory distress syndrome in pregnancy and the puerperium: causes, courses, and outcomes. Obstet Gynecol 2001;97:760-4.

8. Hawkins JL, Koonin LM, Palmer SK, Gibbs CP. Anesthesia-related deaths during obstetric delivery in the United States, 1979-1990. Anesthesiology 1997;86:277-84.

9. Lee W, O'Cornnell CM, Baskett TF. Maternal and perinatal outcomes of eclampsia: Nova Scotia, 1981-2000. 7 Obstet Gynaecol Can 2004;26:119-23.

10. Kieser KE, Baskett TF. A 10-year population-based study of uterine rupture. Obstet Gynecol 2002;100:749-53.

11. Health Canada. Canadian perinatal bealth report, 2003. Ottawa: Minister of Public Works and Government Services Canada; 2003.
12. Commission on Professional and Hospital Activities. International classification of disease, 9th revision: clinical modification. Ann Arbor (Mich.): The Commission; 1992.

13. Canadian Classification of Diagnostic. Therapeutic, and surgical procedures. Ottawa: Statistics Canada; 1986.

14. Huston P, Naylor CD. Health services research: reporting on studies using secondary data sources. CMA7 1996;155(12):1697-709.

Correspondence to: Dr. Shi Wu Wen, Ottawa Hospital,

501 Smyth Rd., Box 241, Ottawa ON K1H 8L6;

fax613 739-6266; swwen@ohri.ca

Appendix 1: Diagnostic ${ }^{12}$ and procedure $\operatorname{codes}^{13}$ used for identifying cases of severe maternal morbidity

ICD-9 codes from the International Classification of Diseases, 9th revision: ${ }^{12}$

- Venus thromboembolism: 673.2

- Eclampsia: 642.6

- Pulmonary, cardiac and CNS complications of anesthesia: 668.0-668.2

- Cerebrovascular disorders in the puerperium, including intracranial venous sinus thrombosis: $674.0,671.5,430-434$ 436-437

- Uterine rupture: 665.0, 665.1

- Adult respiratory distress syndrome: 518.5, 518.81, 518.82

- Pulmonary edema: 518.4, 428.1

- Myocardial infarction: 410, 411

- Acute renal failure after labour and delivery: 669.3

- Cardiac arrest or failure or cerebral anoxia after obstetric surgery: 669.4

- Severe postpartum hemorrhage requiring hysterectomy, transfusion or both:* 666.0-666.3

CCP codes, from the Canadian Classification of Diagnostic, Therapeutic, and Surgical Procedures: ${ }^{13}$

- Ventilation: 13.62, 13.63

- Transfusion (whole blood, packed cells): 13.03, 13.04

- Abdominal hysterectomy: 80.2, 80.3

Note: $\mathrm{CNS}=$ central nervous system

*Used in conjunction with CCP codes $^{13}$ for abdominal hysterectomy or transfusion.

\section{Appendix 2: Diagnostic ICD-9 $\operatorname{codes}^{12}$ used to identify} cases of pre-existing conditions

- Systematic lupus erythematosus: 710.0

- Cystic fibrosis: 227.02

- Chronic renal disease: 581-583, 585, 587, 588

- Essential hypertension: 401

- Diabetes mellitus: 250.0

- Diabetes mellitus with renal or other complications: 250.4250.8

- Pulmonary hypertension: $416.0,416.8$

- Chronic rheumatic heart disease: 393-398

- Ischemic heart disease: 412, 414.8, 414.9 